\title{
Influence of the neck shape for Helmholtz resonators
}

\author{
Jean-François Mercier, ${ }^{1}$ Jean-Jacques Marigo, ${ }^{2}$ and Agnès Maurel ${ }^{3, a)}$ \\ ${ }^{1}$ Poems, CNRS, Ecole Nationale Supérieure des Techniques Avancées ParisTech, INRIA, \\ 828 boulevard des Maréchaux, Palaiseau, 91762, France \\ ${ }^{2}$ Laboratoire de Mécanique du Solide, CNRS, Ecole Polytechnique, Route de Saclay, Palaiseau, \\ 91128 , France \\ ${ }^{3}$ Institut Langevin, CNRS, ESPCI ParisTech, 1, rue Jussieu, Paris, 75005, France
}

(Received 22 August 2017; revised 4 November 2017; accepted 26 November 2017; published online 20 December 2017)

\begin{abstract}
The resonance of a Helmholtz resonator is studied with a focus on the influence of the neck shape. This is done using a homogenization approach developed for an array of resonators, and the resonance of an array is discussed when compared to that of a single resonator. The homogenization makes a parameter $\mathcal{B}$ appear which determines unambiguously the resonance frequency of any neck. As expected, this parameter depends on the length and on the minimum opening of the neck, and it is shown to depend also on the surface of air inside the neck. Once these three geometrical parameters are known, $\mathcal{B}$ has an additional but weak dependence on the neck shape, with explicit bounds. (C) 2017 Acoustical Society of America. https://doi.org/10.1121/1.5017735
\end{abstract}

[MRH]

Pages: 3703-3714

\section{INTRODUCTION}

The vessels named "echea" are probably the first acoustic resonators. Described by Vitruvius in his "De Architectura," echea were used in ancient Greek theaters for their acoustic properties, ${ }^{1}$ and later on in churches and mosques to improve the edifice sonority. ${ }^{2-4}$ This resonator has then been studied in detail by Helmholtz who was mainly interested in its musical properties, ${ }^{5}$ and nowadays, it is the most popular resonator for the acoustic waves used to control and reduce the noise in ducts with flows ${ }^{6}$ or in the design of metasurfaces to realize sound collimators ${ }^{7}$ or sound absorbers. $^{8,9}$

Since Helmholtz's works, many studies have been devoted to this resonator and more or less sophisticated expressions of the resonance frequency have been proposed. Starting with Ingard, ${ }^{10}$ most of these studies use approximate modal methods and they aim to derive the so-called "added lengths" $e_{\mathrm{ad}}$, which accounts for the effect of the evanescent field excited in the vicinity of the resonator neck; see, e.g., Ref. 11. This evanescent field, or near field, affects significantly the scattering properties of resonators with thin necks, with the case of soda cans [with a neck reduced to a zero thickness diaphragm, $e=0$ in Fig. 1(a)] being a borderline case. The added length enters into the expression of the wavenumbers $k_{r}$ at the resonances, with

$$
\operatorname{cotan} k_{r} d=k_{r} H \frac{e+e_{\mathrm{ad}}}{h^{-}},
$$

whose popular expression for the lowest Helmholtz resonance with $k_{r} d \ll 1$ reads as

$$
k_{r}^{2}=\frac{h^{-}}{\left(e+e_{\mathrm{ad}}\right) \mathcal{S}_{c}},
$$

\footnotetext{
${ }^{\text {a)} E l e c t r o n i c ~ m a i l: ~ a g n e s . m a u r e l @ e s p c i . f r ~}$
}

written here in a two-dimensional configuration with $\mathcal{S}_{c}=d H$ the surface of the cavity and $\left(e, h^{-}\right)$the length and the minimum opening of the neck (Fig. 1); see, e.g., Ref. 12, and references therein. Modal methods have been shown to efficiently account for the near field effects for relatively simple geometries. ${ }^{10-13}$ In this study, we are interested in exploring more complex neck shapes and we propose a model able to deal with such complexity. Beyond the fundamental interest of the study, adding extra degrees of freedom in the neck geometry may be of interest for practical applications, including the use of these resonators in metamaterials. ${ }^{7-9}$

A typical question that we have in mind is the following: Consider the four bottles in Fig. 2; can we predict their resonance frequencies or at least predict which ones give similar resonance frequencies? To answer this question, we shall use a homogenization procedure able to encapsulate the effect of the necks in effective jump conditions. The homogenized model applies to an array of resonators producing a resonance frequency comparable to the resonance frequency of a single resonator. This is discussed in Sec. II
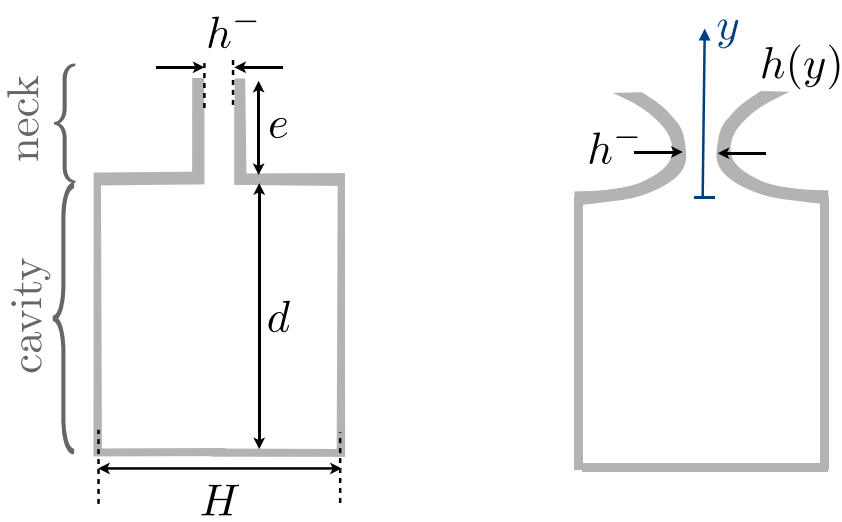

FIG. 1. (Color online) (a) Simple geometry of the neck, for which Eq. (1) applies. (b) Complex geometry of the neck, for which Eq. (3) applies. 


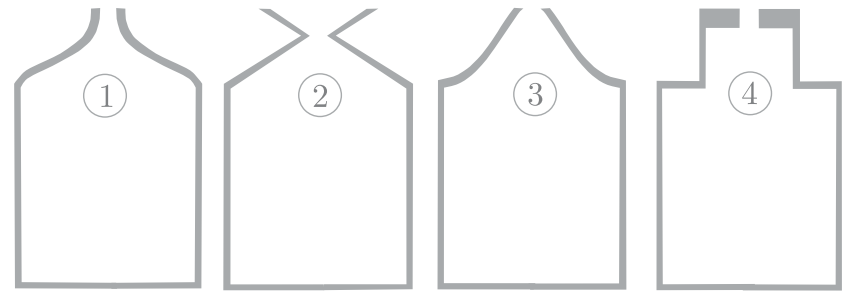

FIG. 2. Can we predict which bottles give similar resonance frequencies?

where the resonances in the homogenized model are found to be the solutions of the dispersion relation

$\operatorname{cotan} k_{r} d=k_{r} H \mathcal{B}$,

where $\mathcal{B}$ depends on the shape of the neck only, and it is the unique quantity needed to determine $k_{r}$. The effective parameter $\mathcal{B}$ is obtained by solving a so-called elementary problem obtained in the homogenization procedure, which corresponds to the problem of a perfect fluid flowing in a duct obstructed by the neck. In this context, $\mathcal{B}$ measures the blockage of the flow due to the presence of this obstacle. Although $\mathcal{B}$ can be calculated by solving numerically the elementary problem, it makes sense to study its dependence with the geometrical parameters of the neck. This is the object of Sec. III; we show that $\mathcal{B}$ is essentially determined by three parameters being the length and the minimum opening of the neck, as expected from Eq. (1), and in addition to the surface of air inside the neck. Once these three parameters are known, $\mathcal{B}$ has an additional but weak dependence on the neck shape. We give an explicit expression of $\mathcal{B}$ for a simple shape formed by two parts with constant opening, a case which appears to be of particular interest, and we provide a procedure to get explicit bounds of $\mathcal{B}$ for an arbitrary neck shape. Finally, we inspect the validity of our homogenized model [Eq. (3)] in Sec. IV in light of comparison with direct numerical calculations of the resonance frequencies.

\section{HELMHOLTZ RESONANCE IN THE HOMOGENIZED PROBLEM}

\section{A. From a single resonator to an array of Helmholtz resonators}

To begin with, we inspect the relation between the resonance of a single resonator and that of a periodic array of resonators, and to make the comparison possible, one has to define what we term resonance. For a single resonator, the resonances refer traditionally to the frequencies producing a peak of velocity in the neck. For an array of resonators, they are defined when the plane on the top of the neck is associated with a Dirichlet boundary condition for the acoustic pressure; see, e.g., Ref. 14, which is consistent with the traditional conception. This condition is termed in-phase reflection in electromagnetism, and, transposed to acoustics, "in-phase" refers to no reflection phase shift of the acoustic velocity. For an open cavity $[h(y)=H$ and $e=0]$, it corresponds to the quarter wavelength criterion with zero and maximum acoustic velocities at the extremities of the cavity.
We computed the resonance of a single resonator and that of arrays of resonators with different array spacings $s$; we used the finite element code $\mathrm{XLiFE}++.{ }^{15}$ We considered a simple rectangular form of the neck as in Fig. 1(a) with $h^{-} / H=0.1, e / H=0.1$, and a cavity length set to $d / H$ $=1.5$. The variations of the pressure reflection coefficient $R$ are reported in Fig. 3 for $s / H=1,3$, and 7 , and the pressure fields at the resonances defined as the in-phase reflection $\operatorname{Re}(R)=-1$ are shown in Figs. 4(a)-4(c). For a single resonator, we computed the mean velocity, averaged on the volume of the neck. It is reported in Fig. 3 as a function of the frequency (dotted lines); the field at the frequency realizing the maximum velocity in the neck is shown in Fig. 4(d). In Fig. 3, we observe a small shift of the resonance frequency of the arrays when increasing $s / H\left(k_{r} H=0.48,0.46\right.$, and $0.44)$ toward the frequency realizing the peak of velocity for the single resonator (at $k_{r} H=0.44$ ). This result is quite intuitive; when they are close to each other, the resonators have interactions that result in a shift of their resonance frequency, and it turns out that this shift goes to higher frequencies. For larger spacings, these collective effects weaken and, eventually, we recover the behavior of a single resonator. It is worth noting that this applies up to $s_{c}=2 \pi / k$ (at normal incidence); indeed, when $s>s_{c}$, higher diffracted waves appear that affect the resonance (see Appendix A). Finally, a small influence of the incidence angle on the resonance frequencies is observed for the arrays; no influence is observed for a single resonator.

From what has been said above, the resonance frequency of an array of resonators does not coincide exactly with that of a single resonator and in fact, for a given shape of the resonator, the resonance of an array is not known precisely until the spacing has been specified. In the following, we shall consider the more compact array with $s=H$, which leads to a simpler effective model and allows us to focus on the effects of the neck only (see Appendix A for $s>H)$.

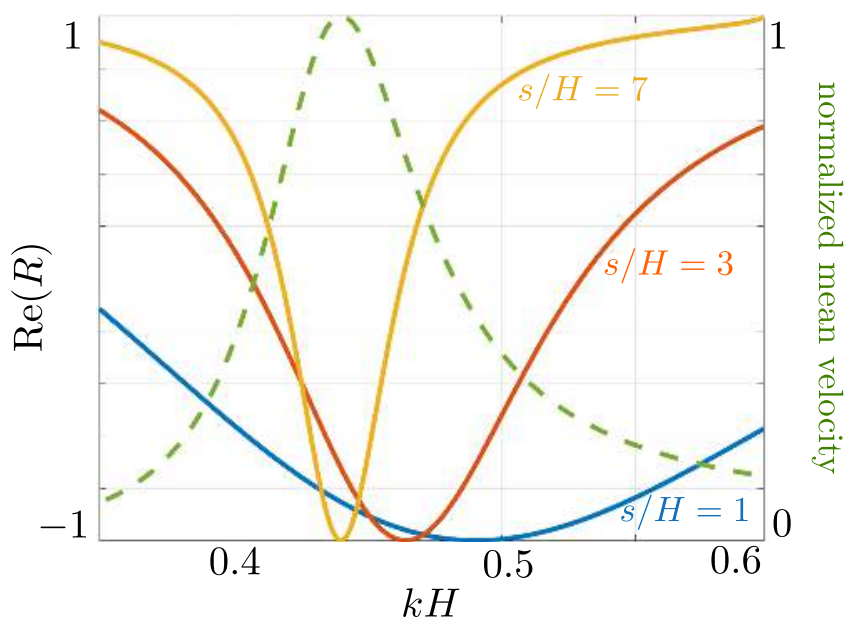

FIG. 3. (Color online) Small shift of the resonance frequency for arrays of Helmholtz resonators with increasing relative spacings $s / H$; the resonances of the arrays are given by the in-phase condition $\operatorname{Re}(R)=-1$ (plain lines). The dotted line shows the variations of the mean velocity in the neck for a single resonator in free space (the mean velocity is normalized to its maximum value). 

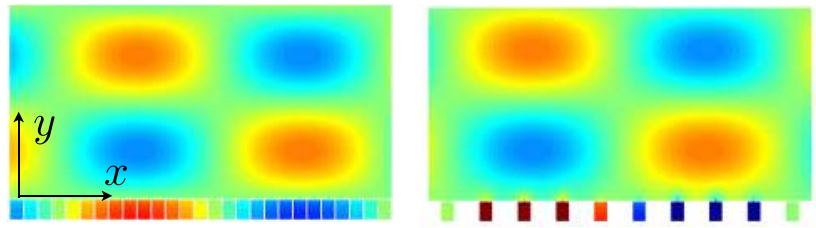

Arrays of resonators: (a) $s / H=1$,

(b) $s / H=3$,

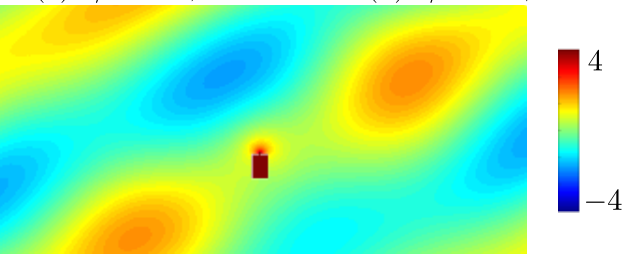

(c) $s / H=7$,
FIG. 4. (Color online) Pressure fields at the resonances (a)-(c) for arrays of resonators with various spacings $s / H$; the incident plane wave of amplitude 1 is at oblique incidence $\theta=30^{\circ}$, (d) for a single resonator in free space for the same incident wave. Each resonator has dimensions $e / H=0.1, \quad h^{-} / H$ $=0.1$, and $d / H=1.5$.

(d) Single resonator in free space

\section{B. Homogenization of an array of Helmholtz resonators}

In the physical problem, the pressure $p(\mathbf{x})$ and velocity $\mathbf{u}(\mathbf{x})$ satisfy the linearized Euler equations

$$
\mathbf{u}=\nabla p, \quad \operatorname{div} \mathbf{u}+k^{2} p=0,
$$

with $\mathbf{x}=(x, y)$, and the condition of vanishing normal velocity applies at each boundary between air and the rigid parts of the resonator. In the homogenized problem, the region of the cavities is simply replaced by air. The region of the neck is assumed to be small compared to that of the cavities and it is replaced by an interface across which jump conditions apply (Fig. 5). Specifically, the pressure field $p(y)$ in the homogenized problem satisfies

$$
\left\{\begin{array}{l}
\partial_{y y} p(y)+k^{2} p(y)=0, \quad-d<y<0, \text { and } y>e, \\
\llbracket p \rrbracket=H \mathcal{B} \overline{\partial_{y} p}, \quad \llbracket \partial_{y} p \rrbracket=-\frac{e H-\mathcal{S}_{\mathrm{n}}}{H} k^{2} \bar{p}, \\
\partial_{y} p(-d)=0,
\end{array}\right.
$$

where for any function $f(y)$, we have defined the jump $\llbracket f \rrbracket$ $\equiv f(e)-f(0)$, and the mean value $\bar{f} \equiv(1 / 2)[f(0)+f(e)]$.
This is obtained because of two simplifications in the physical problem. First and as previously said, we consider a compact array with vanishing thickness of the cavity walls $s=H$. Next, we restrict ourselves to the case of waves at normal incidence on the array. The full calculation has to account, if $s \neq H$, for the thickness of the cavity wall, and, if oblique incidences are considered, for the rigid boundary condition on the cavity walls; see Ref. 16 and Appendix A.

In Eq. (5), a one-dimensional wave equation applies for $-d<y<0$ with a Neumann boundary condition at $y=-d$. This is a classical result from the homogenization at the leading order, and it has been shown that the result holds true at the next order. ${ }^{16}$ In Eq. (5), the jump conditions are a less classical result; we shall derive below these conditions for an incident wave at normal incidence.

The Helmholtz resonance being subwavelength, we have $k e, k H \ll 1$. For simplicity, we set $\varepsilon=H \ll 1$ with $k$ $=O(1)$ and $e=O(H)$. Following Ref. 16, we also use $d$ $=O(1)$ and we define two expansions in the near and far fields of the necks, specifically

$$
\left\{\begin{array}{l}
p=p^{0}(y)+\varepsilon p^{1}(y)+\cdots, \quad \mathbf{u}=u_{y}^{0}(y) \mathbf{e}_{y}+\varepsilon u_{y}^{1}(y) \mathbf{e}_{y}+\cdots, \quad \text { in the far field } \\
p=q^{0}(\boldsymbol{\xi})+\varepsilon q^{1}(\boldsymbol{\xi})+\cdots, \quad \mathbf{u}=\mathbf{v}^{0}(\boldsymbol{\xi})+\varepsilon \mathbf{v}^{1}(\boldsymbol{\xi})+\cdots, \quad \text { in the near field }
\end{array}\right.
$$
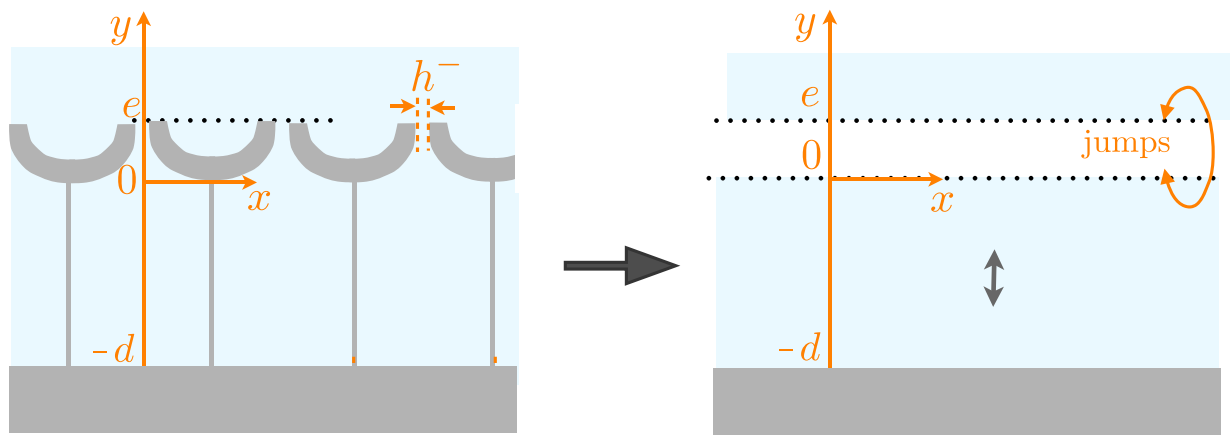

FIG. 5. (Color online) From the actual problem to the homogenized problem. In the homogenized problem, the neck is replaced by a region across which jump conditions apply, Eq. (5). 
with $\boldsymbol{\xi}=\mathbf{x} / \varepsilon$, and $\boldsymbol{\xi}=\left(\xi_{x}, \xi_{y}\right)$. In the far field, we used that $\partial / \partial_{x}=0$, thus, the problem is one-dimensional along $y$. Obviously, this applies to the far field only; the near field, in the vicinity of the necks, contains the evanescent field which is two-dimensional in $\xi$ coordinates. Eventually, the near field is simply periodic with respect to $\xi_{x}$ and of period unity in this rescaled coordinate. More specifically, the inner region is reduced to a strip $\mathrm{Y}_{\infty}=\{(-1 / 2,1 / 2)$ $\times(-\infty, \infty)\} \backslash S_{n}$ where $S_{\mathrm{n}}$ is the region occupied by the rigid parts of the neck; we shall use also $Y=\{(-1 / 2,1 / 2)$ $\left.\times\left(-\xi_{m}, \xi_{m}\right)\right\} \backslash S_{n}\left(\mathrm{Y}_{\infty}\right.$ corresponds to $\mathrm{Y}$ for $\left.\xi_{\mathrm{m}} \rightarrow+\infty\right)$.
From a unique problem, we have built two problems, in the near and far fields, and we have to specify the boundary conditions for each one. The boundary conditions on the rigid parts of the necks apply to the near field solution, but boundary conditions are missing when $\xi_{y} \rightarrow \pm \infty$. Reversely, the radiation conditions apply to the far field solution but there are missing boundary conditions when approaching the necks $y \rightarrow 0^{ \pm}$. These missing boundary conditions are provided simultaneously by the matching conditions, which read as

$$
\left\{\begin{array}{l}
p^{0}\left(0^{ \pm}\right)=\lim _{\xi_{y} \rightarrow \pm \infty} q^{0}(\boldsymbol{\xi}), \quad \mathbf{u}^{0}\left(0^{ \pm}\right)=\lim _{\xi_{y} \rightarrow \pm \infty} \mathbf{v}^{0}(\boldsymbol{\xi}), \\
p^{1}\left(0^{ \pm}\right)=\lim _{\xi_{y} \rightarrow \pm \infty}\left(q^{1}(\boldsymbol{\xi})-\xi_{y} \partial_{y} p^{0}\left(0^{ \pm}\right)\right), \quad \mathbf{u}^{1}\left(0^{ \pm}\right)=\lim _{\xi_{y} \rightarrow \pm \infty}\left(\mathbf{v}^{1}(\boldsymbol{\xi})-\xi_{y} \partial_{y} \mathbf{u}^{0}\left(0^{ \pm}\right)\right) .
\end{array}\right.
$$

The above conditions tell us that the near and far fields have to coincide in some intermediate region, say at a distance $\sqrt{\varepsilon}$ of the necks, and there $y \sim \sqrt{\varepsilon} \rightarrow 0^{ \pm}$and $\xi_{y}=y / \varepsilon \rightarrow \pm \infty$. To derive the jump conditions that interrogate the far field solution for $y \rightarrow 0^{ \pm}$, we shall use the expansions (6) in Eq. (4) with the differential operator $\nabla$ $\rightarrow \mathbf{e}_{y} \partial_{y}$ in the far field and $\nabla \rightarrow(1 / \varepsilon) \nabla_{\xi}$ in the near field.

Let us start at the leading order. In the inner region, this leading order is in $1 / \varepsilon$ in Eq. (4). We get $\nabla_{\xi} q^{0}=0$ from which $q^{0}$ is constant. The matching condition $(7)$ at the order 0 allows to conclude that $p^{0}\left(0^{ \pm}\right)=q^{0}$ and, thus, that $p^{0}$ is continuous at $y=0$. Also, we get

$$
\operatorname{div} \xi \mathbf{v}^{0}=0, \quad \lim _{\xi_{y} \rightarrow \pm \infty} \mathbf{v}^{0}=u_{y}^{0}\left(0^{ \pm}\right) \mathbf{e}_{y}
$$

which shows that $\mathbf{v}^{0}$ is symmetric with respect to $\xi_{x}$ $\in(-1 / 2,1 / 2)$ in view of the symmetry of the problem; therefore, the periodic boundary conditions are equivalent to rigid boundary conditions $\mathbf{v}^{0} \cdot \mathbf{n}=0$. To end with the leading order, we integrate the relation $\operatorname{div} \xi \mathbf{v}^{0}=0$ over $Y_{\infty}$; using the rigid boundary conditions on the neck boundary and at $\xi_{x}= \pm 1 / 2$, we get that $v_{y}^{0}\left(\xi_{x},+\infty\right)=v_{y}^{0}\left(\xi_{x},-\infty\right)$. It is now sufficient to use the matching condition to conclude that $u_{y}^{0}$ is continuous at $y=0$. The jump conditions at the leading order correspond to the usual continuity conditions of the pressure and the normal velocity. In the present case, these conditions are clearly unsatisfactory since they ignore the effect of the neck.

To capture the effect of the evanescent field in the vicinity of the necks, we have to go to the next order. To do so, we consider the problem satisfied by $q^{1}$ in the near field region, with $\mathbf{v}^{0}=\nabla_{\xi} q^{1}$ in Eq. (8), and the boundary conditions $\mathbf{v}^{0} \cdot \mathbf{n}=0$ on the boundaries of the neck and at $\xi_{x}= \pm 1 / 2$. This problem is linear with respect to $\partial_{y} p^{0}(0)$ $=u_{y}^{0}\left(0^{ \pm}\right)$so that we can write

$$
q^{1}(\boldsymbol{\xi})=\partial_{y} p^{0}(0) \hat{q}(\boldsymbol{\xi})+Q,
$$

with $Q$ a constant and $\hat{q}(\xi)$ satisfying

$$
\Delta_{\xi} \hat{q}=0, \quad \lim _{\xi_{y} \rightarrow \pm \infty} \nabla_{\xi} \hat{q}=\mathbf{e}_{y}
$$

and the same boundary conditions apply to $\hat{q}$ as to $q^{1}$. This is the elementary problem that will provide the parameter $\mathcal{B}$ involved in the jump conditions. Specifically, from Eq. (10), $\left(\hat{q}-\xi_{y}\right)$ tends to constant values $\hat{\mathcal{B}}^{ \pm}$when $\xi_{y} \rightarrow \pm \infty$. Accordingly, the field $\hat{q}$ reads as

$$
\left\{\begin{array}{l}
\hat{q}(\xi)=\xi_{y}+\hat{\mathcal{B}}^{-}+q^{\mathrm{ev}}(\xi), \quad \xi_{y}<0, \\
\hat{q}(\xi)=\xi_{y}+\hat{\mathcal{B}}^{+}+q^{\mathrm{ev}}(\xi), \quad \xi_{y} \geq 0
\end{array}\right.
$$

where $q^{\text {ev }}$ is an evanescent field vanishing at $\xi_{y} \rightarrow \pm \infty$.

The jump of $p^{1}$ immediately follows owing to Eq. (7) along with Eqs. (9) and (11); we get

$$
p^{1}\left(0^{+}\right)-p^{1}\left(0^{-}\right)=\hat{\mathcal{B}} \partial_{y} p^{0}(0)
$$

with $\hat{B}=\hat{B}^{+}-\hat{B}^{-}$. To get the jump of $u_{y}^{1}$, we shall integrate over $\mathrm{Y}$ the relation $\operatorname{div} \xi \mathbf{v}^{1}+k^{2} p^{0}(0)=0$ [from Eq. (4), where we used that $q^{0}=p^{0}(0)$, which we obtained at the leading order]. Using the Neumann boundary conditions on the boundaries of the rigid parts and at $\xi_{x}= \pm 1 / 2$, we get $\int_{Y} \mathrm{~d} \xi \operatorname{div} \xi \mathbf{v}^{1}=v_{y}^{1}\left(\xi_{x},+\xi_{m}\right)-v_{y}^{1}\left(\xi_{x},-\xi_{m}\right)$. We also have $\int_{Y} \mathrm{~d} \xi p^{0}(0)=p^{0}(0)\left(2 \xi_{m}-\mathcal{S}_{n} / H^{2}\right)$, where $\mathcal{S}_{n}$ is the surface of the neck. With $\xi_{m} \rightarrow+\infty$, it is sufficient to use the matching condition (7) and the relation $\partial_{y} u_{y}^{0}+k^{2} p^{0}=0$, from Eq. (4), to get $u_{y}^{1}\left(0^{+}\right)-u_{y}^{1}\left(0^{-}\right)=\left(\mathcal{S}_{n} / H^{2}\right) k^{2} p^{0}(0)$.

The final jump conditions will be written after two last steps. First, we shall express the jump conditions across an enlarged version of the interface; for the time being, they have been written across $y=0^{ \pm}$, thus, reducing the regions of the necks to a zero thickness interface. Next, we shall write these jumps on a unique homogenized field $p^{h}$, which 
admits the same expansion in $\varepsilon$ as $\left(p^{0}+\varepsilon p^{1}\right)$, thus, the same expansion as $p$, up to $O\left(\varepsilon^{2}\right)$. To do so, we use that $p^{0}(e)=p^{0}\left(0^{+}\right)+e \partial_{y} p^{0}(e)+O\left(e^{2}\right)$. With $e=O(\varepsilon)$, we get

$$
\begin{aligned}
\llbracket p^{0}+\varepsilon p^{1} \rrbracket & =e \partial_{y} p^{0}(e)+\varepsilon\left(p^{1}\left(0^{+}\right)-p^{1}\left(0^{-}\right)\right)+O\left(\varepsilon^{2}\right) \\
& =(e+H \hat{\mathcal{B}}) \overline{\partial_{y} p^{0}}+O\left(\varepsilon^{2}\right) \\
& =(e+H \hat{\mathcal{B}}) \overline{\partial_{y}\left(p^{0}+\varepsilon p^{1}\right)}+O\left(\varepsilon^{2}\right),
\end{aligned}
$$

since $p^{0}\left(0^{+}\right)-p^{0}\left(0^{-}\right)=0$ and $\overline{\partial_{y} p^{0}}=\partial_{y} p^{0}(e)+O(\varepsilon)$ [and the same with $\left.\partial_{y} p^{0}(0)\right]$. Doing the same for $\left(u_{y}^{0}+\varepsilon u_{y}^{1}\right)$, it is easy to see that $p^{h}$ satisfying (i) $\partial_{y y} p^{h}+k^{2} p^{h}=0$ outside the enlarged interface $y \in(0, e)$, and (ii) the jumps across the enlarged interface $\llbracket p^{h} \rrbracket=(e+H \hat{\mathcal{B}}) \overline{\partial_{y} p^{h}}$ and $\llbracket \partial_{y} p^{h} \rrbracket$ $=-\left(e-\mathcal{S}_{n} / H\right) k^{2} p^{h}$, admits the same expansion as $\left(p^{0}\right.$ $\left.+\varepsilon p^{1}\right)$ up to $O\left(\varepsilon^{2}\right)$, thus, the same expansion of $p$ up to $O\left(\varepsilon^{2}\right)$. We recover Eq. (5) with $\mathcal{B}=e / H+\hat{\mathcal{B}}$.

Note that we can derive jump conditions involving other values of $\mathcal{B}_{a}=a / H+\hat{\mathcal{B}}$ across interfaces enlarged to a thickness $a=O(e)$ (this excludes values of $a$ on the order of d). The resulting models are all equivalent up to $O\left(\varepsilon^{2}\right)$ but may slightly differ in terms of accuracy; see Refs. 17 and 18.

\section{Resonance frequency in the homogenized problem}

The homogenized problem (5) is one-dimensional, and it is easy to solve it for an incident plane wave along $y$. The solution reads as

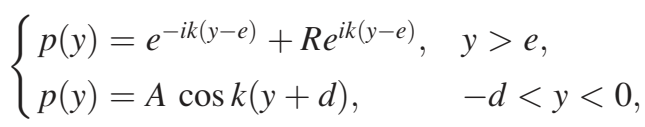

where the boundary condition $\partial_{y} p(-d)=0$ has been accounted for. The reflection coefficient $R$ and the constant $A$ are determined by the jump conditions in Eq. (5), which allows us to determine the wavenumbers $k_{r}$ at the resonances defined by $R=-1$, solutions of the dispersion relation

$$
\mathcal{B} k_{r} H \tan k_{r} d+\frac{k_{r}{ }^{2}}{4} \mathcal{B}\left(e H-\mathcal{S}_{n}\right)=1 .
$$

Owing to the fact that the contribution in $k_{r}^{2}$ in the righthand side term is small compared to $1\left[k_{r}{ }^{2} e H=O\left(\varepsilon^{2}\right)\right]$, we get Eq. (3): $\operatorname{cotan} k_{r} d=k_{r} H \mathcal{B}$.

\section{THE BLOCKAGE COEFFICIENT IN THE ELEMENTARY PROBLEM}

From Eq. (3), it is sufficient to compute $\mathcal{B}$ to get the resonance frequency, and we shall see in Sec. III A that this is true. Thus, it makes sense to inspect in some detail the variation of $\mathcal{B}$ with the neck shapes in order to draw the tendencies that may help us to design the necks for a given purpose. To that aim, we shall first see some qualitative properties of $\mathcal{B}$. Then, we shall consider in detail the case of a neck composed of two-stepwise constant parts, hereafter termed two-step neck, for which an excellent approximate expression of $\mathcal{B}$ is available. Besides, it appears that such simple neck, with 2 degrees of freedom (in addition to $h^{-} / H$ and $e(H)$, describe the whole accessible space for $\mathcal{B}$. This means that a neck with any complicated shape profile has the same resonance frequency (because the same $\mathcal{B}$ ) than a twostep neck. Based on this fact, we provide bounds for $\mathcal{B}$ for an arbitrary neck shape.

\section{A. Some qualitative properties of $\mathcal{B}$}

The elementary problem (10), which provides $\mathcal{B}=\hat{\mathcal{B}}$ $+e / H$, is a static problem, which corresponds to a perfect fluid flowing in a duct obstructed by the neck (Fig. 6). In general, this problem has to be solved numerically, but we can already anticipate which neck geometries will produce high or low $\mathcal{B}$ values. With $\hat{p}$ the velocity potential, the constant $\hat{\mathcal{B}}$, often termed blockage coefficient, measures the deviation of the streamlines with respect to the straight lines that we would have in an empty duct. As a first remark, the flow being reversible, the neck can be upturned without affecting the value of $\mathcal{B}$, a fact of interest when one considers Helmholtz resonators with necks inserted partially or totally inside the cavity. ${ }^{13,19,20}$

Next, increasing $e / H$ or decreasing $h^{-} / H$ produces an increasing blockage of the flow, whence an increase in $\mathcal{B}$. In the absence of neck $\hat{\mathcal{B}}=0$ and it is positive, otherwise; this can be already seen from Eq. (1) with $\mathcal{B} \geq e / H$.

Finally, the regions where the fluid is at rest are of particular interest. Indeed, they behave as rigid parts, being delimited by a streamline with vanishing normal velocity, by definition. As such, starting from a neck producing such regions, we can build a family of necks with the same $\mathcal{B}$ by replacing these regions, partially or totally, by rigid parts. We expect the fluid to be at rest in the region of surface $\mathcal{S}_{e}$ in Fig. 6; it is the outer region of the neck through which the flow cannot pass and inside which it will not enter deeply. We have checked this property on $\mathcal{B}$, and illustrations will be given in Sec. III B.

From what has been said above, we expect that only the inner surface $\mathcal{S}$ of air in the neck, corresponding to the region where the fluid can flow, influences the value of $\mathcal{B}$. From now on, and to avoid heavy notations, we define

$$
\varphi_{n}=\frac{\mathcal{S}}{e H}, \quad \alpha^{-}=\frac{h^{-}}{H},
$$

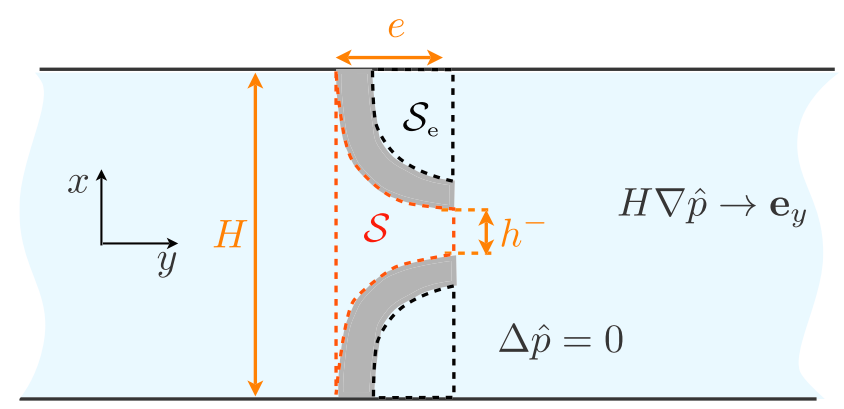

FIG. 6. (Color online) Elementary problem in physical scales, with $\hat{p}(\mathbf{x})$ $=\hat{q}(\xi)$ the velocity potential associated to a flow in a duct obstructed by an obstacle. The obstacle is characterized by its length $e$ and the minimum relative opening $h^{-}=\alpha^{-} H$. The perfect fluid flows through a region of surface $\mathcal{S}=\varphi_{n} e H$ (inner region) within the neck. The outer region $\mathcal{S}_{e}$ roughly corresponds to a region where the flow is at rest. 
being, respectively, the inner filling fraction of air and the relative minimum opening of the neck (whence by construction $0 \leq \alpha^{-} \leq 1$ and $\left.\alpha^{-} \leq \varphi_{n} \leq 1\right)$.

\section{B. The case of two-step neck shapes}

We term two-step neck shape a neck made of two rectangular parts with for any $0<\beta<1$,

$$
\left\{\begin{array}{l}
h(y)=\alpha^{+} H, \quad 0 \leq y<\beta e \\
h(y)=\alpha^{-} H, \quad \beta e<y \leq e
\end{array}\right.
$$

see the inset of Fig. 7. Extending the approximate expression of $\mathcal{B}$ given in Ref. 17 for a one-step rectangular neck, it appears that $\mathcal{B}$ is accurately described by the expression

$$
\mathcal{B}=\frac{e}{H}\left[\frac{(1-\beta)}{\alpha^{-}}+\frac{\beta}{\alpha^{+}}\right]-\frac{1}{\pi} \log \left(\sin \frac{\pi \alpha^{-}}{2} \sin \frac{\pi \alpha^{-}}{2 \alpha^{+}} \sin \frac{\pi \alpha^{+}}{2}\right) .
$$

In addition to $e / H$ and $\alpha^{-}$, this family of necks has two other degrees of freedom being $\alpha^{+} \in\left(\alpha^{-}, 1\right)$ and $\beta \in(0,1)$ the relative opening and relative thickness of the largest part of the neck, respectively. Next, the inner fraction of air in the neck is given by

$$
\varphi_{n}=\alpha^{-}(1-\beta)+\alpha^{+} \beta .
$$

There are two contributions in the expression of $\mathcal{B}$, Eq. (18). The first contribution, proportional to $e / H$ is related to the propagation of the low frequency wave; it would be obtained by approximate modal methods only accounting for the propagating wave and, as such, appears as a simple extension of Eq. (1) with $e_{\mathrm{ad}}=0$. We term this contribution the propagation contribution. The second contribution in $\log$ is well known in the case of a single diaphragm with zero thickness

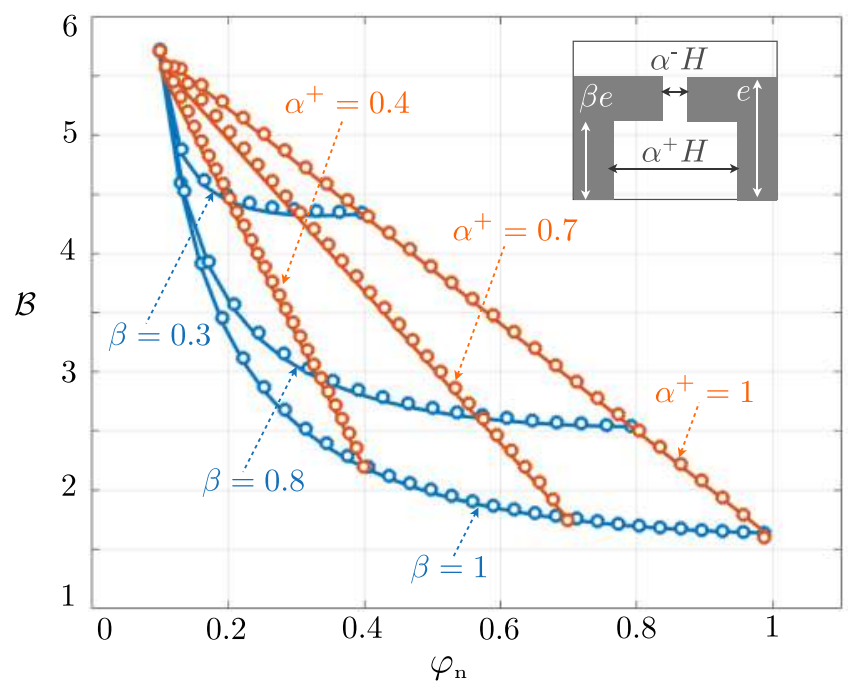

FIG. 7. (Color online) Variation of $\mathcal{B}$ for fixed values of $\alpha^{-}=0.1$ and $e / H=0.45$ as a function of $\varphi_{n}$, Eq. (19), for a two-step neck shape (shown in the inset). $\mathcal{B}$ describes the interior of a wing for varying $\alpha^{+}$and $\beta$ according to Eq. (18) (plain lines); the open symbols show the actual values of $\mathcal{B}$ from the resolution of the elementary problem, Eqs. (10) and (11). The wing shaped is bounded by $\mathcal{B}_{M}$ for $\alpha^{+}=1$ and $\mathcal{B}_{m}$ for $\beta s=1$, Eq. (20). (the derivation using techniques of complex variables can be found in Ref. 21). It is, for instance, the unique contribution to $\mathcal{B}$ for a soda can resonator; in this case, with $e=0$, there is no propagation contribution and with $\alpha^{+}=\alpha^{-}$the diaphragm only produces a near field contribution [with $\mathcal{B}=-2$ / $\left.\pi \log \sin \left(\pi \alpha^{-} / 2\right)\right]$. In Eq. (18), we assume that the expression of the near field contribution holds true at each discontinuity in the neck cross section; next, using the reversibility of the flow, the contribution is written in order that either an expansion or a contraction produces an increase in $\mathcal{B}$ (thus, with an argument in each sine being smaller than $\pi / 2$ ).

Inspecting the variations of $\mathcal{B}$ with $\alpha^{+}$and $\beta$ reveals that $\mathcal{B}$ can take any value between two extreme curves parametrized by $\varphi_{n}: \mathcal{B}_{M}$ corresponding to $\alpha^{+}=1$ (a single step shape) and $\mathcal{B}_{m}$ corresponding to $\beta=1$ (a diaphragm with opening $\alpha^{-}$ staked to a single step). These two extrema are given by

$$
\left\{\begin{array}{l}
\mathcal{B}_{m}=\frac{e}{H} \frac{1}{\varphi_{n}}-\frac{1}{\pi} \log \left[\sin \left(\frac{\pi \alpha^{-}}{2}\right) \sin \left(\frac{\pi \varphi_{n}}{2}\right) \sin \left(\frac{\pi \alpha^{-}}{2 \varphi_{n}}\right)\right], \\
\mathcal{B}_{M}=\frac{e}{H}\left[1+\frac{1-\varphi_{n}}{\alpha^{-}}\right]-\frac{2}{\pi} \log \sin \left(\frac{\pi \alpha^{-}}{2}\right),
\end{array}\right.
$$

and they coincide at their minimum and maximum for $\varphi_{n}=1$ and $\varphi_{n}=\alpha^{-}$, respectively, with

$$
\left\{\begin{array}{l}
\min \left(\mathcal{B}_{M}\right)=\min \left(\mathcal{B}_{m}\right)=\frac{e}{H}-\frac{2}{\pi} \log \left(\sin \frac{\pi \alpha^{-}}{2}\right) \\
\max \left(\mathcal{B}_{M}\right)=\max \left(\mathcal{B}_{m}\right)=\frac{e}{h^{-}}-\frac{2}{\pi} \log \left(\sin \frac{\pi \alpha^{-}}{2}\right)
\end{array}\right.
$$

and these bounds are given once $\alpha^{-}=h^{-} / H$ and $e / H$ have been chosen.

To illustrate what has been said above, Fig. 7 reports the variations of $\mathcal{B}$ computed numerically by solving the elementary problem, Eqs. (10) and (11) (open symbols). We considered fixed values of $\alpha^{-}=h^{-} / H=0.1$ and $e / H$ $=0.45$ and varied continuously $\varphi_{n}$. For comparison, we reported $\mathcal{B}$ predicted by Eq. (18) (plain lines). Expectedly, $\mathcal{B}$ describes the interior of a wing shaped region, bounded by Eq. (20) and with extreme values equal to 1.63 and 5.71 in agreement with Eq. (21). As we announced, the accuracy of Eq. (18) is excellent. Note that once $\left(\alpha^{-}, e / H, \varphi_{n}\right)$ are given, $\mathcal{B}$ is determined and it corresponds to a unique neck shape, from Eqs. (18) and (19). This is of importance if we have in mind the inverse problem in which we want to determine the neck able to produce a given resonance frequency with geometrical constraints (the constraints are, for instance, the values of $e$ / $h$ and $\alpha^{-}$that we keep constant).

We shall see in Sec. III C that the bounds (20) and (21) seem to hold for any neck shapes being described by an arbitrary neck shape $h(y)$ once $\left(\alpha^{-}, e / H\right)$ are given, and we shall propose a procedure to get more precise bounds.

\section{Arbitrary neck shape}

We now move on to the case of necks with arbitrary shapes being described by $h(y)$ and the corresponding local opening and inner surface 

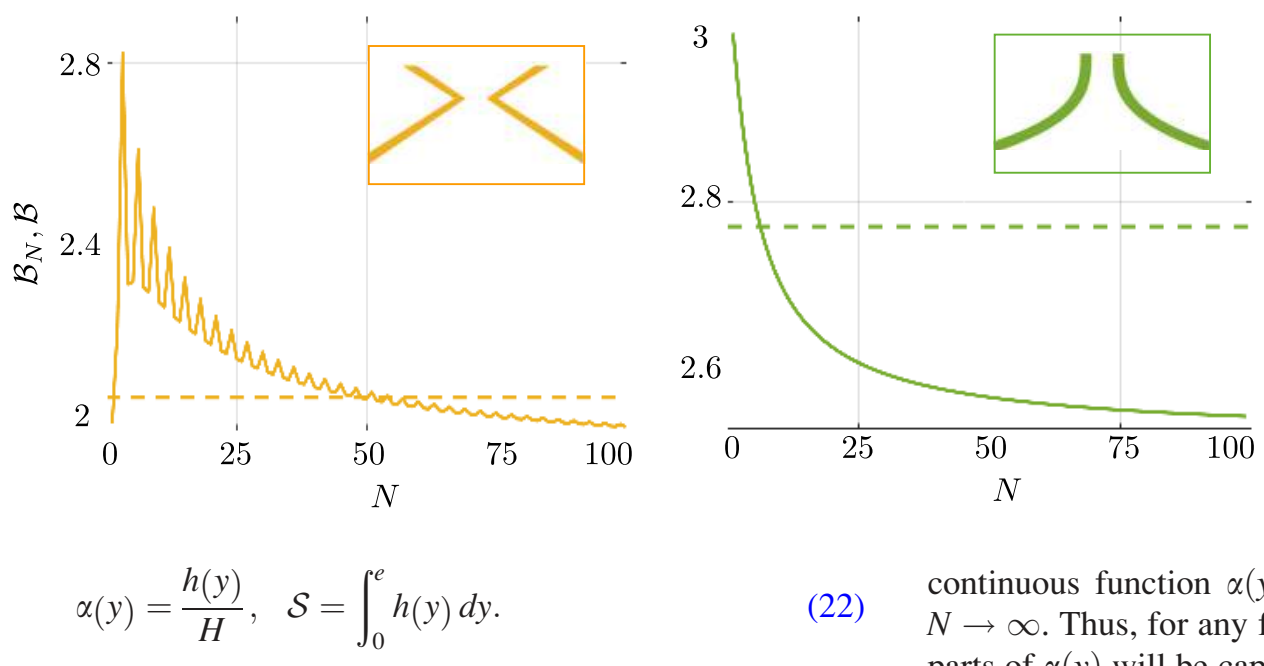

For a two-step neck shape, we identified in Eq. (18) the propagation contribution and the near field contribution. Our guess is that the value of $\mathcal{B}$ for an arbitrary neck shape reads as

$$
\mathcal{B}=\int_{0}^{e} \frac{d y}{h(y)}-\frac{1}{\pi} \log \left(F(\alpha) \sin \frac{\pi \alpha(e)}{2}\right) .
$$

In the above expression, the integral is the continuous version of the propagation contribution in Eq. (18). In the second term, which encapsulates the near field contribution, we accounted for the diaphragm effect at the end of the neck, which is the rule more than the exception [if it is not the case, $\alpha(e)=1$ and this contribution vanishes]. Next, we ask the function $F(\alpha)$ to account for all the near field effects in the neck for $0 \leq y<e$. Obviously, this contribution cannot be evaluated in an arbitrary case; this is why $\mathcal{B}$ has to be calculated numerically by solving Eq. (10) with Eq. (11). However, it is possible to find bounds for $F(\alpha)$ if we admit that any discontinuity in the neck between $\alpha(y)$ and $\alpha(y+$ $\delta y$ ) produces a contribution of the form

$$
\cdots \sin \frac{\pi \alpha(y)}{2 \alpha(y+\delta y)} \cdots,
$$

which is written here for an expansion [the case of a constriction involves $\pi \alpha(y+\delta y) / 2 \alpha(y)$ in the argument of the sine]. In fact, the easiest way to find such bounds, which does not mean that it is the most precise, is as follows. We discretize the interval $(0, e)$ into $N$ parts with $\delta y=e / N$, producing a set of $\left\{\alpha_{i}\right\}_{i=0, \ldots, N}$ with $\alpha_{i}=\alpha(i \delta y)$ (and $\alpha_{0}=1$ at $y=0$ by construction). Doing so, we can construct $F_{N}(\alpha)$ such that

$$
F_{N}(\alpha)=\prod_{i=0}^{N} \sin \frac{\pi \alpha_{i}}{2 \alpha_{i+1}}
$$

written here for a series of expansions with $\alpha_{i+1}>\alpha_{i}$; in the more general case, one choose the ratio of $\min \left(\alpha_{i}, \alpha_{i+1}\right)$ and $\max \left(\alpha_{i}, \alpha_{i+1}\right)$ for each $i$. It is worth noting that an alternative choice would be to fix $\delta \alpha$ with a resulting variable $\delta y$.

In any case, $F_{\infty}>F_{N}$ which means that using $F=F_{\infty}$ in Eq. (23) defines a lower bound for $\mathcal{B}$. Indeed, for a continuous function $\alpha(y), F_{\infty}=1$ since $\alpha_{i} / \alpha_{i+1} \rightarrow 1$ when $N \rightarrow \infty$. Thus, for any function $\alpha(y)$, only the discontinuous parts of $\alpha(y)$ will be captured in this limit. However, evanescent waves are excited in the physical problem even for a continuous profile and this cannot be estimated within the present procedure.

An upper bound is more difficult to define and other constructions of the $F_{N}$ could be chosen depending on the considered shape. As the simplest rule, we consider the maximum of $F_{N}$ when varying $N$. By construction, we find that $N=1$ maximizes $F_{N}$ if $\alpha(e)=\alpha^{-}$, otherwise, the maximum is found for $N$ equal to a few unities.

For several neck shapes, we calculated $\mathcal{B}_{N}$ using $F=F_{N}$ in Eq. (23) varying $N$ and the exact value of $\mathcal{B}$ solving the elementary problem (10) and (11). Typical variations of $\mathcal{B}_{N}$ are reported in Fig. 8 in plain lines, with extreme values bounding, as expected, the actual value of $\mathcal{B}$ (in dotted line).

Doing so for two families of profiles, we obtain the representation of Fig. 9 (the parametrization of these profiles are given in Appendix B). As for the two-step necks, we have imposed the same values of $\alpha^{-}=h^{-} / H$ and of $e / H$. These calculations seem to show that $\mathcal{B}$ lies inside the wing region defined by the two-step necks.

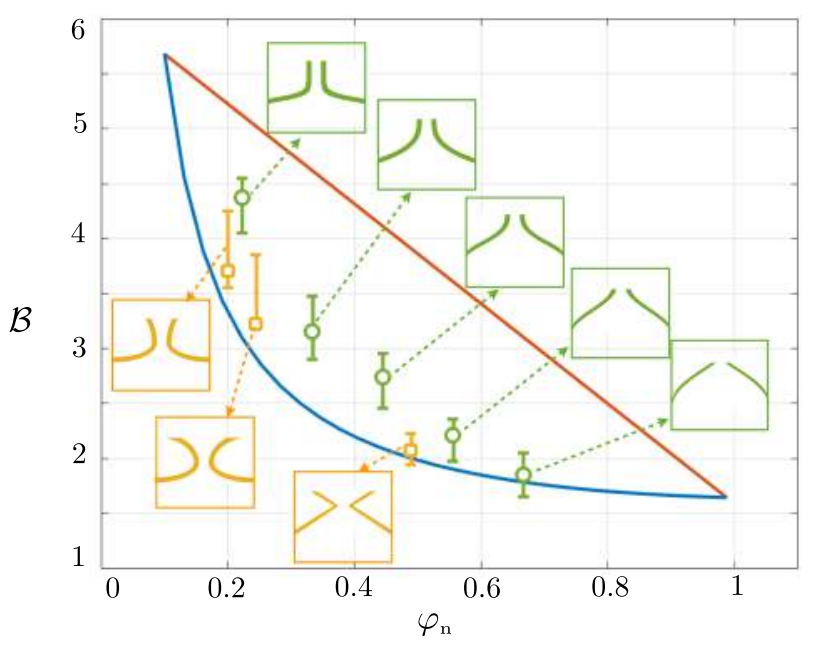

FIG. 9. (Color online) Examples of $\mathcal{B}$ values for continuously varying neck shapes calculated by solving the elementary problems, Eqs. (10) and (11) (open symbols; the insets show the corresponding shapes, all with $\alpha^{-}=0.1$ and $e / H=0.45$ ); the bars around each symbol show the bounds of $\mathcal{B}$, from the procedure illustrated in Fig. 8. The plain lines are the bounds for $\mathcal{B}$ from Eq. (20). 

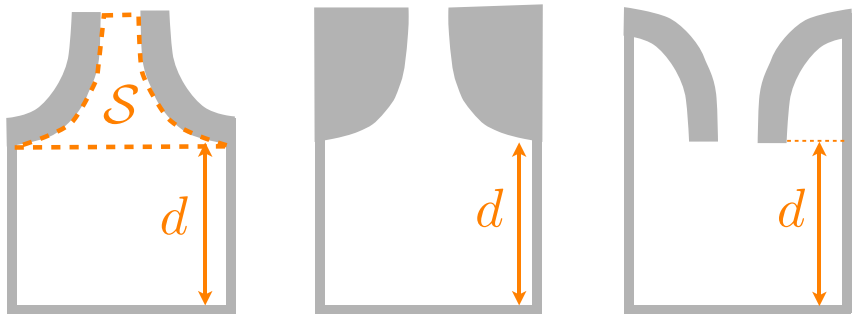

FIG. 10. (Color online) Helmholtz resonators with necks with the same shape $h(y)$ and (a) a finite wall thickness leaving an outer region filled with air, (b) an outer region filled with rigid material, and (c) an upturned neck.

\section{VALIDATION OF THE HOMOGENIZED MODEL FOR THE HELMHOLTZ RESONANCE}

Having drawn the tendencies and the variations of $\mathcal{B}$ as a function of the three main geometrical parameters $\left(h^{-} / H, e / H, \varphi_{n}\right)$ and as a function of the shape, it remains to be seen whether the Eq. (3) is able to predict the frequency of the Helmholtz resonance.

\section{A. Upturned and filled necks}

We start with the properties announced for $\mathcal{B}$ : we said that (i) the region of the neck through which the fluid cannot flow (in the elementary problem) can be filled with rigid material without affecting $\mathcal{B}$, whence without affecting the resonance, and (ii) the neck can be upturned without affecting the resonance frequency, Fig. 10.

We have computed $\mathcal{B}$ for the two shapes of the necks reported in the insets in Figs. 11(a) and 11(c). Evidently, $\mathcal{B}$ is found to be the same for a given neck and its upturned version. Next, for the one-step neck in Fig. 11(a), we found $\mathcal{B}=$ 5.67 for an outer part of the neck being filled with air and $\mathcal{B}=5.71$ for an outer part of the neck being filled with a rigid material. For the continuously varying neck shape in

(a)

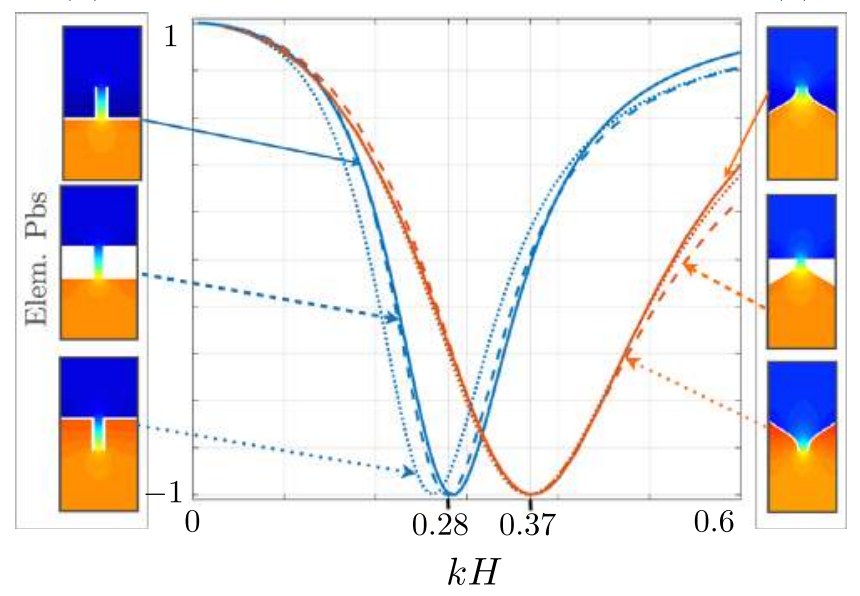

FIG. 11. (Color online) (a), (c) Fields of the velocity potential in the elementary problem Eq. (10) from which $\mathcal{B}$ is deduced, for a neck with an outer part filled with air (top), filled with a rigid material (center), and upturned (bottom); two neck shapes are considered. (b) Real parts of the reflection coefficients calculated numerically. The resonance frequencies are in good agreement with Eq. (3), which gives for (a) $k_{r} H \simeq 0.28$ and for (c) $k_{r} H \simeq 0.37$

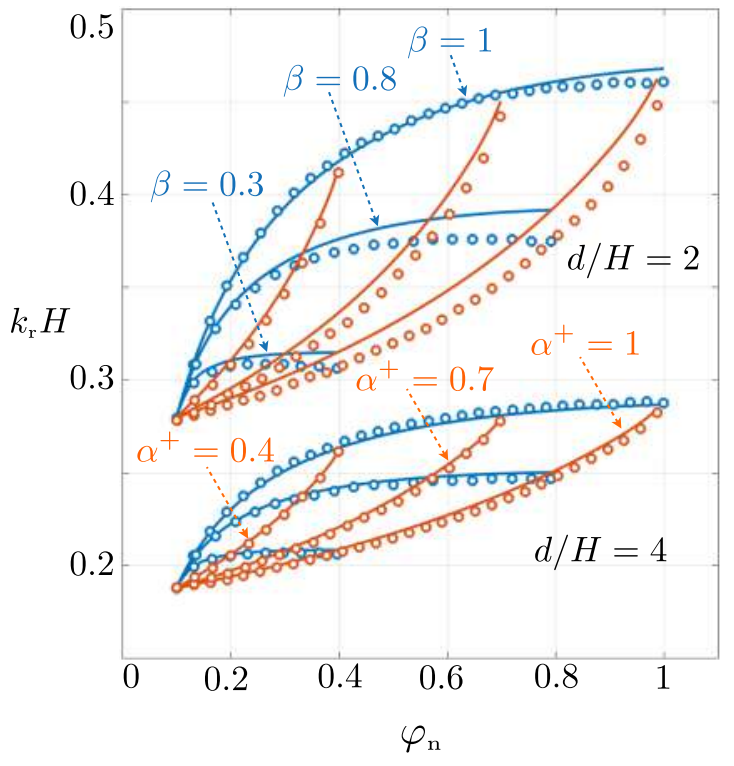

FIG. 12. (Color online) Resonance frequencies as a function of $\varphi_{n}$ for twostep necks (same representation as in Fig. 7) surmounting a cavity with $d / H=2$ and $d / H=4$. Open symbols show the resonance frequencies calculated numerically, and plain lines report the homogenized prediction Eq. (3) using $\mathcal{B}$ in Eq. (18).

Fig. $11(\mathrm{c})$, we found $\mathcal{B}=2.88$ and $\mathcal{B}=2.93$. As expected, the values are close to each other in both cases.

Next, we computed numerically the resonance curves for these necks surmounting a cavity of length $d / H=2$; results are shown in Fig. 11(b). We observe that the resonance frequencies of cavities surmounted by the three necks are very close, as expected. Besides, these resonance frequencies are in good agreement with Eq. (3), which gives for (a) $k_{r} H \simeq 0.28$ and for (c) $k_{r} H \simeq 0.37$.

\section{B. Resonance frequencies in the actual and homogenized problems}

We computed numerically the reflection curve as a function of $k H$ for two-step necks producing the values of $\mathcal{B}$ given in Fig. 7. For each curve, we stored the resonance frequency $k_{r} H$ realizing in-phase reflection, $\operatorname{Re}(R)=-1$. The results are reported in Fig. 12 together with the homogenized prediction (3) using the estimate $(18)$ for $\mathcal{B}$. We considered the neck surmounting a cavity with lengths $d / H=2$ and $d / H=4$. The agreement is, in general, good, which confirms the accuracy of the homogenized model. However, it is visible that it is much better for the largest cavity length $d / H=4$. The relative disagreement between the actual resonance frequencies and the homogenized prediction for $d / H=2$ is attributable to two effects, which are difficult to separate. On the one hand, a smaller cavity depth $d$ produces a higher resonance frequency $k_{r} H$, which eventually exits from the range of validity of the homogenization since we assumed $k H \ll 1$. On the other hand, we have assumed that propagation effect occurs in the cavity, with $d \gg H, e$, and this penalizes also values of $d / H$ of the order 1 .

The same representation is used in Fig. 13 for the necks with continuously varying shape shown in Fig. 9. We reported the resonance frequencies $k_{r} H$ computed numerically (open symbols) and the bars being deduced from Eq. 


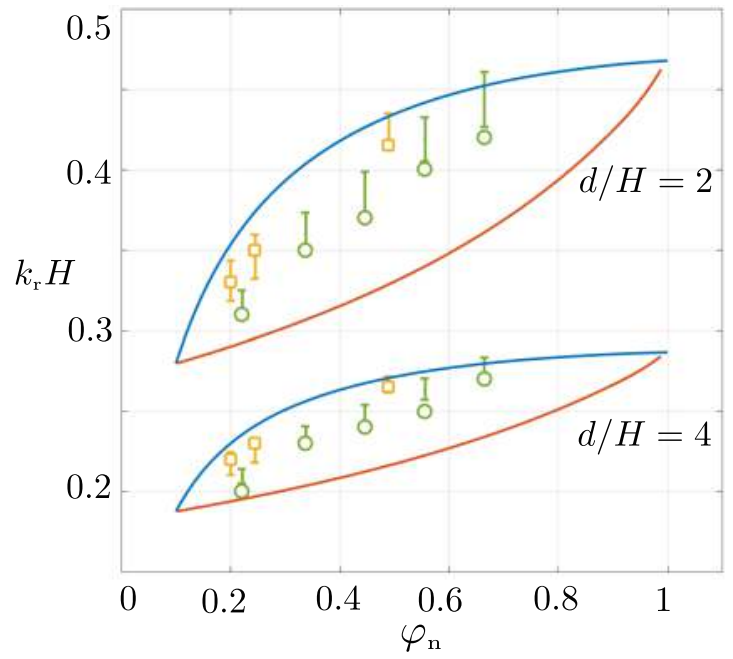

FIG. 13. (Color online) Same representation as in Fig. 12 for continuous varying neck shapes (the necks are the same as in Fig. 9). Open symbols show the resonance frequencies calculated numerically and bars the bounds deduced from $\mathcal{B}_{N}$ (see Fig. 9) in Eq. (3). The plain lines report the bounds of $k_{r} H$ for $\mathcal{B}$ in Eq. (20).

(3) with the values of $\mathcal{B}_{N}$ reported in Fig. 9 [from Eq. (23) with Eq. (25)]. The conclusion is the same as for the two-step shapes with an overall good agreement, and the procedure to get approximate values of $\mathcal{B}$ and, thus, to get the resonance frequency of an arbitrary neck shape appears to be satisfactory. As expected, all the resonance frequencies are bounded by the resonance frequencies given by Eq. (3) with $\left(\mathcal{B}_{m}, \mathcal{B}_{M}\right)$ in Eq. (20) (plain lines).

Finally, the range of accessible $k_{r} H$ values for varying shape decreases when $d$ increases, with a limit given by the asymptotic behavior $k_{r}=\pi /(2 d)$ for large $d$ value.

\section{CONCLUDING REMARKS}

We have presented a homogenization procedure able to describe the scattering properties of arrays of Helmholtz resonators with arbitrary neck shape. In the resulting homogenized problem, the resonances are known unambiguously once a coefficient $\mathcal{B}$ has been calculated, Eq. (3). This coefficient is the blockage coefficient of the simple potential flow problem of a fluid flowing in a duct obstructed by the resonator neck.

If a direct problem is thought where a given neck is considered, we have shown that the three geometrical parameters $\left(e / H, \alpha^{-}, \varphi_{n}\right)$ already provide bounds $\left(\mathcal{B}_{m}, \mathcal{B}_{M}\right)$ of $\mathcal{B}$, Eq. (20), therefore they provide bounds for the resonance frequencies $k_{r} H$, with Eq. (20) in Eq. (3). Then, more precise bounds are possible considering the variations of $\mathcal{B}_{N}$ in the procedure presented in Sec. III.

If an inverse problem is thought where a neck producing a given resonance frequency is looked for, the family of the two-step necks is of particular interest. Indeed, this family has the huge advantage to have a closed form of $\mathcal{B}$, Eq. (18), which implies a few degrees of freedom. Moreover, focusing on this family is not restrictive since it seems that any neck shape has a two-step alter ego producing the same resonances; see Fig. 14 with the illustrative examples of our study. Notably, we illustrated that $\mathcal{B}$ has still some flexibility when geometrical constraints are imposed. We have considered the two most usual constraints on the compactness of the array given by $(e+d)$ and on the minimum opening $h^{-}$, which controls the main source of viscous dissipation (and this later effect can be unwanted for acoustic purpose or desired for application to perfect absorption devices). Incidentally, these shapes are also easy to manufacture compared to their continuously varying counterparts.

We end these concluding remarks by giving the response to our question in Fig. 2, which is basically the kind of response given in Fig. 14. In Fig. 2, the necks have the same $e / H=0.45, \alpha^{-}=0.1$, and $\varphi_{n}=0.2$, and they differ only by their shapes under these constraints. Next, computing $\mathcal{B}$ gives $\mathcal{B} \simeq 2.7$ for the profiles 1 and 4 and $\mathcal{B} \simeq 2.1$ for the profiles 2 and 3 , which allows us to conclude.

\section{ACKNOWLEDGMENTS}

We thank the anonymous referee for his very helpful comments and suggestions. The authors acknowledge the financial support of the French Mission Interdisciplainare du Centre National de la Recherche Scientifique (MI/CNRS) under Grant No. INFYNITI/PomS. (a)

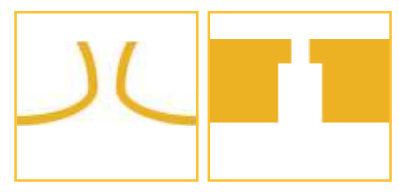

(e)

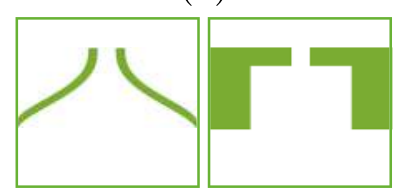

(b)

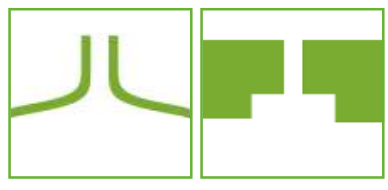

(f)

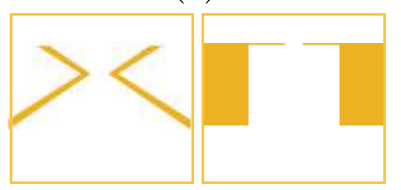

(c)

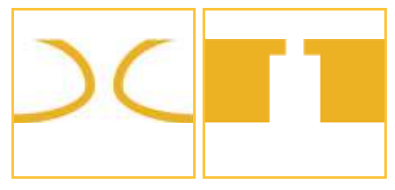

$(\mathrm{g})$

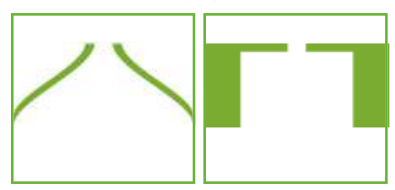

(d)

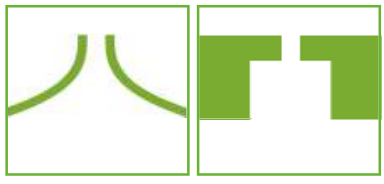

(h)

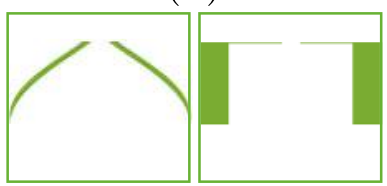

FIG. 14. (Color online) Equivalence between a smoothly varying profile and a two-step shape with the same $\left(e / H, \alpha^{-}, \varphi_{n}\right)$ and $\mathcal{B}$. For each couple (a)-(h), on the left a profile from Fig. 9 and on the right the corresponding two-step shape providing the same resonance frequencies. (a) $\varphi_{n}=0.20$, (b) $\varphi_{n}=0.22$, (c) $\varphi_{n}=0.24$, (d) $\varphi_{n}=0.33$, (e) $\varphi_{n}=0.44$, (f) $\varphi_{n}=0.49$, (g) $\varphi_{n}=0.56$, and (h) $\varphi_{n}=0.67$ (for $\mathcal{B}$, see Fig. 9). 


\section{APPENDIX A: RESONANCE CURVES IN THE HOMOGENIZED PROBLEM}

In Fig. 3, we reported resonance curves calculated numerically. Then only the resonance frequencies deduced from these numerical curves and that given by Eq. (3) in the homogenized problems were compared, and we restricted ourselves to the case $s / H=1$. For the sake of completeness, we report in this appendix comparisons of the entire resonance curves.

When the thickness of the wall does not vanish $(s / H>1)$ and at oblique incidence, the jump conditions read

$$
\llbracket p \rrbracket=s \mathcal{B} \bar{v}, \quad \llbracket v \rrbracket=-s \mathcal{C} \frac{\partial^{2} \bar{p}}{\partial x^{2}}-e \varphi_{n} k^{2} \bar{p},
$$

with $\llbracket v \rrbracket=v(x, e)-v(x, 0)$ the jump of the normal component of the acoustic velocity (along $y$ ), but now $v(x, e)$ $=\partial_{y} p(x, e)$ and $v(0)=\alpha_{c} \partial_{y} p(x, 0)$, with

$$
\alpha_{c}=\frac{H}{s} \text {. }
$$

This is because the region of the cavities is now replaced by an effective anisotropic medium with the acoustic velocity $\mathbf{u}=(u, v)$ with $u=0$ and $v=\alpha_{c} \partial_{y} p$; this tells us that the propagation is allowed along $y$ only, as expected. In addition, we have $\operatorname{div} \mathbf{u}+k^{2} \alpha_{c} p=0$, from which the propagation is described by the wave equation $\partial_{y y} p+k^{2} p=0$ (with the same wavenumber as in air). Thus, in Eq. (A1), $s$ appears explicitly in the right-hand side terms, and it appears implicitly in the left-hand side terms since $\llbracket v \rrbracket$ and $\bar{v}$ depend on the filling fraction $\alpha_{c}$ of air within the cavities. Next, $\mathcal{B}$ is affected as well since the flow in the elementary problem (10) [see also Eq. (11)], now takes place in a duct of varying section: it is of width $\alpha_{c}$ in the inner region $\xi_{y}<0$ (with $\xi_{y}=y / s$ ) and of width 1 for $\xi_{y}>e / s$. As in Eq. (5) and according to Eq. (16), the parameter $\varphi_{n}$ is defined by

$$
\varphi_{n}=\frac{e s-\mathcal{S}_{n}}{e s}
$$

being the filling fraction of air in the neck (for a simple neck shape as in Fig. 15, $\varphi_{n}=\alpha$ ). Finally, $\mathcal{C}$ is a new parameter in Eq. (A1), which is involved in the jump conditions only if $p$ depends on $x$, that is, for oblique incidence. For the one-step neck in Fig. 15, we have good estimates of $(\mathcal{B}, \mathcal{C})$ in the forms

$$
\left\{\begin{array}{l}
\mathcal{B}=\frac{e}{\alpha s}-\frac{1}{\pi} \log \left(\sin \frac{\pi \alpha}{2} \sin \frac{\pi \alpha}{2 \alpha_{c}}\right), \\
\mathcal{C}=\frac{e \alpha}{s}-\frac{\pi \alpha^{2}}{4} .
\end{array}\right.
$$

We recover Eq. (18) for $H=s$ (zero wall thickness) and $\alpha$ $=\alpha^{-}=\alpha^{+}$(one-step neck).

In the following, we considered $H=1, \alpha=0.1, \alpha_{c}=1$, $e=0.1$, and $s=1,3,7$.

Computing $\mathcal{B}$ and $\mathcal{C}$ by means of the elementary problems, we find the parameters entering in Eq. (A1): $s \mathcal{B}=2.2$, $7.7,19.8$ and $s \mathcal{C}=0.0020,0.0007,0.0003$, respectively. Our

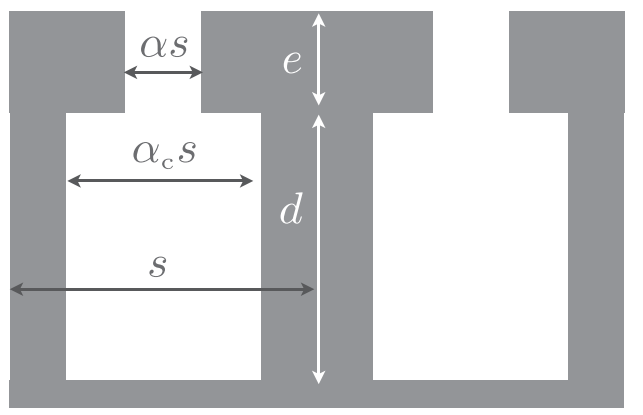

FIG. 15. Array of Helmholtz resonators with nonvanishing thickness of the cavity walls $(s / H>1)$.

estimates (A4) give $s \mathcal{B}=2.2,7.6$, and 19.6 and $s \mathcal{C}=0.0021$, 0.0007 , and 0.0003. Finally, $e \varphi_{n}=0.01000 .00330 .0014$. The same coefficients apply for any cavity depth $d$.

For an oblique incidence $\theta$, we look for a solution of the form

$$
\left\{\begin{array}{l}
p(y)=e^{i k_{x} x}\left(e^{-i k_{y}(y-e)}+R e^{i k_{y}(y-e)}\right), \quad y>e \\
p(y)=A e^{i k_{x} x} \cos k(y+d), \quad-d<y<0
\end{array}\right.
$$

with $k_{x}=k \cos \theta, k_{y}=k \sin \theta$. The reflection coefficient in the homogenized problem then reads as

$$
\left\{\begin{array}{l}
R=-\frac{Z}{Z^{*}}, \text { with } Z=z_{1} y_{1}-z_{2} y_{2} \\
z_{1}=1+i \frac{\mathcal{B}}{2} k_{x} s, \quad z_{2}=i k_{x}+\frac{1}{2}\left(\mathcal{C} k_{y}^{2} s-\frac{\alpha_{c}}{s} k^{2} e\right) \\
y_{1}=\frac{k \alpha_{c}}{s} \tan (k d) \frac{1}{2}-\left(\mathcal{C} k_{y}^{2} s-\frac{\alpha_{c}}{s} k^{2} e\right) \\
y_{2}=1-\frac{\mathcal{B}}{2} k \alpha_{c} \tan (k d) .
\end{array}\right.
$$

Figure 16 reports the variations of $R$ calculated numerically and already shown in Fig. 3 (open symbols in Fig. 16). The results given by the homogenized prediction (A6) are reported in plain lines. We considered incidence angles $\theta=0^{\circ}, 30^{\circ}$, and $60^{\circ}$ and cavity depths $d=1.5$ and $d=6$ (resulting in $k_{r} H \simeq 0.44$ and $k_{r} H \simeq 0.19$, respectively). For $d=6$, the agreement is good for the three spacing and incidences. For $d=1.5$, the agreement remains relatively good but fails for $\theta=60^{\circ}$ and $s / H=7$; in this case, the mode -1 realizing $k_{-1} s=2 \pi /(1+\sin \theta)$ appears for $k<k_{-1}=0.48$ at $\theta=60^{\circ}$, which makes the homogenized prediction unreliable.

From the inspection of these results, the homogenized model is unexpectedly robust and the reason remains to be clarified. Indeed, the small parameter in the asymptotic analysis is now $k s$. Thus, for $s / H=7$, the prediction is correct while $k s$ has largely overcame 1 . To understand such robustness, it would be helpful to conduct the homogenization at higher orders, and to determine the scaling of the higher order effective parameters, say $\mathcal{B}_{i}$ for $i>1$ (with $\mathcal{B}=\mathcal{B}_{1}$ ); if $\mathcal{B}_{i}$ is sufficiently small in a sense that remains to be determined, we could have $\varepsilon^{i} \mathcal{B}_{i} \ll \varepsilon \mathcal{B}$ even for $\varepsilon=k s>1$. 

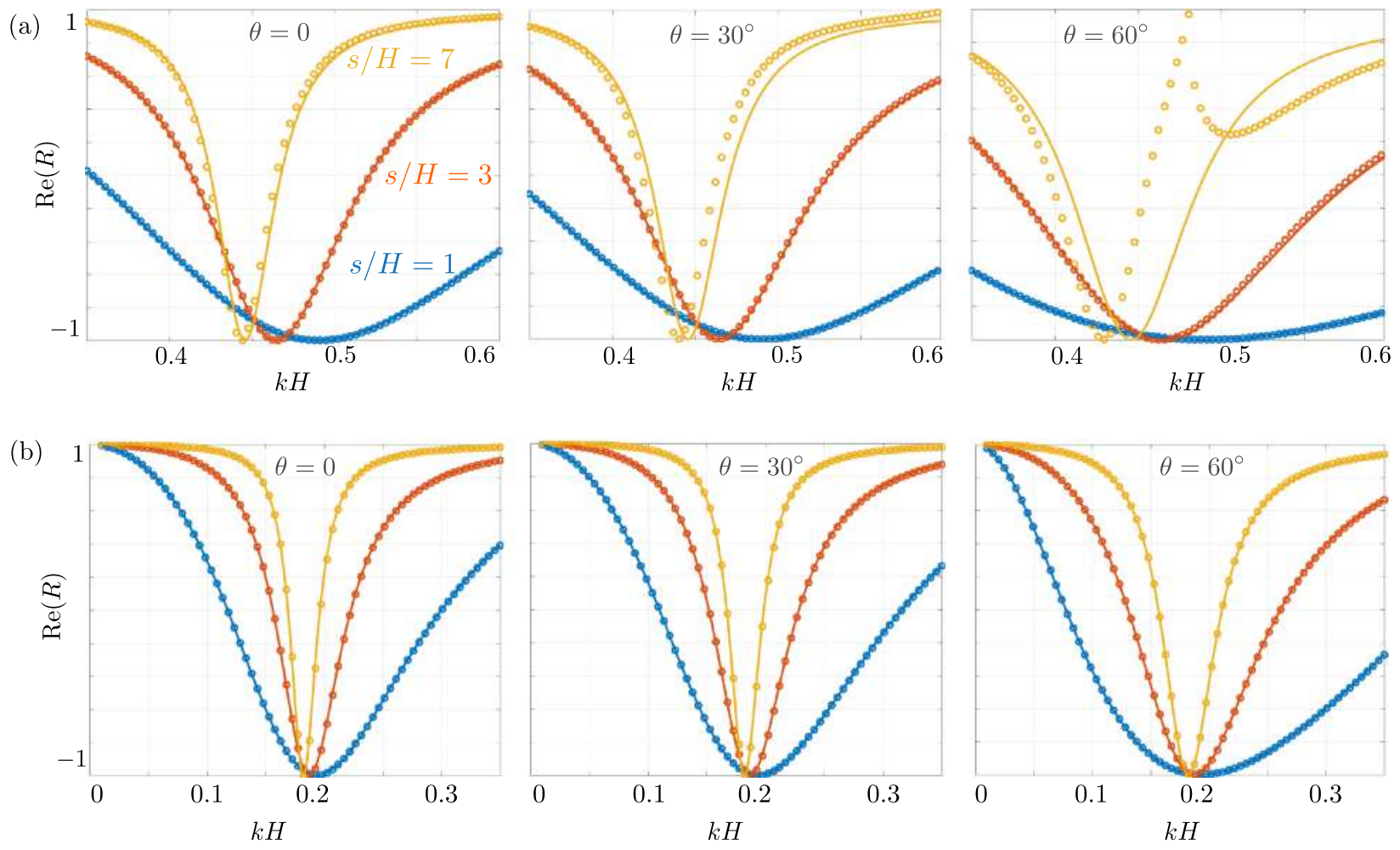

FIG. 16. (Color online) Comparison of the resonance curves, obtained from numerics (symbols) and from the homogenized model, Eq. (A6), for the same geometry of the necks as in Fig. 3, (a) $d=1.5$ and (b) $d=6$.

\section{APPENDIX B: CONTINUOUSLY VARYING SHAPE- CHOICE OF THE PARAMETRIZATION}

The first family of shapes are parametrized by two parameters $(a, b)$

$$
\begin{aligned}
& y=0.45-t, \\
& x=A\left[1-\left(\frac{t}{A}\right)^{b}\right]^{a},
\end{aligned}
$$

and

$$
\begin{cases}t \in(0.40,0), & A=0.40 \\ t \in(0,0.45), & A=0.45\end{cases}
$$

In practice, $(a, b)$ are on the order of unity (the circles in Fig. 9).

The second family of shapes are parametrized by four parameters $(A, T, a, b)$ with

$$
\begin{aligned}
& y=t_{1}-t, \\
& x=A\left[1-\left(\frac{t}{T}\right)^{b}\right]^{a},
\end{aligned}
$$

and

$$
\left\{\begin{array}{l}
t \in\left(0, t_{1}\right), \quad A=0.45, \quad T=t_{1}, \\
t \in\left(t_{1}-0.45,0\right), \quad A=0.45, \quad T=t_{1}-t_{2} \\
t \in\left(t_{1}-0.05,0\right), \quad A=0.40, \quad T=t_{1}-0.05 \\
t \in\left(0, t_{1}-0.45\right), \quad A=0.40, \quad T=t_{1}-t_{2}+0.45
\end{array}\right.
$$

In practice, $(A, T, a, b)$ are on the order of unity (the squares in Fig. 9).

${ }^{1}$ S. Polychronopoulos, D. Kougias, P. Polykarpou, and D. Skarlatos, "The use of resonators in ancient Greek theatres," Acta Acust. united Acust. 99(1), 64-69 (2013).

${ }^{2}$ T. Zakinthinos and D. Skarlatos, "The effect of ceramic vases on the acoustics of old Greek orthodox churches," Appl. Acoust. 68(11), 1307-1322 (2007).

${ }^{3}$ J.-C. Valière, B. Palazzo-Bertholon, J.-D. Polack, and P. Carvalho, "Acoustic pots in ancient and medieval buildings: Literary analysis of ancient texts and comparison with recent observations in french churches," Acta Acust. united Acust. 99(1), 70-81 (2013).

${ }^{4} \mathrm{M}$. R. Ismail, "A parametric investigation of the acoustical performance of contemporary mosques," Front. Architect. Res. 2(1), 30-41 (2013).

${ }^{5} \mathrm{H}$. Helmholtz and A. J. Ellis, On the Sensations of Tone as a Physiological Basis for the Theory of Music, edited by A. J. Ellis (Dover, New York, 1954).

${ }^{6} \mathrm{~W}$. Bi, V. Pagneux, D. Lafarge, and Y. Aurégan, "An improved multimodal method for sound propagation in nonuniform lined ducts," J. Acoust. Soc. Am. 122(1), 280-290 (2007).

${ }^{7}$ L. Quan, F. Qian, X. Liu, X. Gong, and P. A. Johnson, "Mimicking surface plasmons in acoustics at low frequency," Phys. Rev. B 92(10), 104105 (2015).

${ }^{8}$ N. Jiménez, W. Huang, V. Romero-García, V. Pagneux, and J.-P. Groby, "Ultra-thin metamaterial for perfect and quasi-omnidirectional sound absorption," Appl. Phys. Lett. 109(12), 121902 (2016).

${ }^{9}$ V. Romero-García, G. Theocharis, O. Richoux, A. Merkel, V. Tournat, and V. Pagneux, "Perfect and broadband acoustic absorption by critically coupled sub-wavelength resonators," Sci. Rep. 6, srep19519 (2016).

${ }^{10} \mathrm{U}$. Ingard, "On the theory and design of acoustic resonators," J. Acoust. Soc. Am. 25(6), 1037-1061 (1953).

${ }^{11}$ J. Kergomard and A. Garcia, "Simple discontinuities in acoustic waveguides at low frequencies: Critical analysis and formulae," J. Sound Vib. 114(3), 465-479 (1987).

${ }^{12} \mathrm{R}$. Chanaud, "Effects of geometry on the resonance frequency of Helmholtz resonators," J. Sound Vib. 178(3), 337-348 (1994).

${ }^{13}$ A. Selamet and I. Lee, "Helmholtz resonator with extended neck," J. Acoust. Soc. Am. 113(4), 1975-1985 (2003). 
${ }^{14}$ L. Quan, X. Zhong, X. Liu, X. Gong, and P. A. Johnson, "Effective impedance boundary optimization and its contribution to dipole radiation and radiation pattern control," Nature Commun. 5, 3188 (2014).

${ }^{15}$ http://uma.enstaparistech.fr/soft/XLiFE++/ (Last viewed December 12, 2017).

${ }^{16}$ J.-J. Marigo and A. Maurel, "Second order homogenization of subwavelength stratified media including finite size effect," SIAM J. Appl. Math. 77(2), 721-743 (2017).

${ }^{17}$ J.-J. Marigo and A. Maurel, "Homogenization models for thin rigid structured surfaces and films," J. Acoust. Soc. Am. 140(1), 260-273 (2016).
${ }^{18}$ J.-J. Marigo, A. Maurel, K. Pham, and A. Sbitti, "Effective dynamic properties of a row of elastic inclusions: The case of scalar shear waves," J. Elastic. 128(2), 265-289 (2017).

${ }^{19} \mathrm{Z}$. Kang and Z. Ji, "Acoustic length correction of duct extension into a cylindrical chamber," J. Sound Vib. 310(4), 782-791 (2008).

${ }^{20} \mathrm{P}$. Chaitanya and M. Munjal, "Effect of wall thickness on the end corrections of the extended inlet and outlet of a double-tuned expansion chamber," Appl. Acoust. 72(1), 65-70 (2011).

${ }^{21} \mathrm{P}$. M. Morse and K. U. Ingard, Theoretical Acoustics (Princeton University Press, Princeton, NJ, 1968), p. 486. 